\title{
A lattice Boltzmann model for heat transfer in porous media
}

\author{
Sheng Chen ${ }^{* 1,2,3}$ Bo Yang ${ }^{1}$ Chuguang Zheng ${ }^{1}$ \\ 1. State Key Laboratory of Coal Combustion, Huazhong University of Science and \\ Technology, Wuhan 430074, China \\ 2. Institute for Modelling and Simulation in Fluodynamics, Nanoscience and \\ Industrial Mathematics "Gregorio Millán Barbany", Universidad Carlos III de \\ Madrid, Leganes 28911, Spain \\ 3. Faculty of Engineering, The University of Nottingham, University Park, \\ Nottingham NG7 2RD, UK \\ * Corresponding author. Faculty of Engineering, University of Nottingham. E-mail \\ address: shengchen.hust@gmail.com
}

\begin{abstract}
Porous media are commonly found not only in the nature but also in industries. Furthermore, porous media is an important research prototype for a diversity of disciplines. So far a REV (representative elementary volume) scale lattice Boltzmann (LB) model has been proposed and popularly used for investigation on heat transfer in porous media. Unfortunately, such model suffers from a serious drawback that it can not address an investigated domain where the heat capacitance (the product of density and specific heat capacity) of porous media varies spatially obviously. Such deficit restricts dramatically its applicable range. The purpose of the present work is to remedy such serious shortcoming in a simple way. Numerical validation demonstrates the capability and reliability of the present model. In order to clearly show the advantage of the present model, here a single-relaxation-time LB model is taken as an example to illustrate how to remedy the shortcoming of previous models. Its multiple-relaxation-time counterpart can be established straightforwardly in the same way.
\end{abstract}

Key words: Lattice Boltzmann method; heat transfer; porous media

\section{Introduction}

Fluid saturated porous media exist popularly in the nature, such as soil and fractured rocks. Many man-made materials (e.g. cements and metal foams) 
can be considered as porous media, too. Especially, a lot of biological tissues, like bones and wood, can be treated as porous media rationally. Consequently, saturated porous media, as an important research prototype, are commonly found in a wide range of disciplines, for example, manufacture industry, geology, food engineering, bioscience, etc. [1]. Generally, for numerical research, porous media can be modelled at three levels. Along the direction from fine to coarse-gain level, they read [2]: pore-scale, the representative elementary volume (REV) scale, and the domain scale. Considering the balance between necessary information and computational cost, the REV scale description is the most popular one adopted in engineering research [3].

During the past three decades, the lattice Boltzmann (LB) method has attracted increasing attention due to its intrinsic advantages, such as relatively easy treatment of complicated geometry and high parallel computing efficiency[4]. Originally, the LB method was adopted as a powerful tool for pore-scale modelling of porous media [5-7]. The first REV scale LB model for isothermal fluid saturated porous media was proposed by Guo and Zhao [8]. Soon, the same authors designed a thermal LB model for simulation of heat transfer in porous media [9]. Due to its simplicity and reliability, their REV scale LB model has been widely used for research on heat transfer in porous media, such as phase change in porous media $[10,11]$, natural convection in porous media [12] and convective heat transfer in a channel partially filled by porous media [13]. In order to improve the numerical stability of Guo's thermal LB porous media model, which is based on the so-called single-relaxation-time (SRT) approximation, a multiple-relaxation-time (MRT) counterpart has been constructed by Liu et al. [10,14]. Recently, Wang et al. [15] introduced a lattice kinetic SRT scheme to improve the numerical stability of Guo's model.

Guo's thermal LB porous media model is based on the assumption of local equilibrium between saturating fluid and solid matrix, where heat transfer behavior is governed by $[9-15]$

$$
\sigma \partial_{t} T+\nabla_{\alpha} T u_{\alpha}=\nabla_{\alpha} \kappa_{m} \nabla_{\alpha} T
$$

In Eq.(1), $u_{\alpha}$ and $T$ are the volume-averaged velocity and temperature of fluid in the saturated porous media, respectively. The parameter $\sigma=\epsilon+(1-$ $\epsilon)\left(\rho C_{p}\right)_{s} /\left(\rho C_{p}\right)_{f}$ denotes the ratio of heat capacitance (the product of density and specific heat capacity) between saturated porous media and saturating fluid, where $\epsilon$ is the porosity of the porous media, and $\left(\rho C_{p}\right)_{s}$ and $\left(\rho C_{p}\right)_{f}$ are the heat capacitance of solid matrix and of saturating fluid, respectively. In addition, $\kappa_{m}=\lambda /\left(\rho C_{p}\right)_{f}$ is the effective thermal diffusivity, where $\lambda$ is the effective thermal conductivity and $\left(\rho C_{p}\right)_{f}$ is the heat capacitance of saturating fluid.

Recently, several thermal LB models for non-equilibrim heat transfer in fluid 
saturated porous media were published [16,17]. As the governing equation of energy in a non-equilibrium status $[16,17]$ is completely different from its local-equilibrium counterpart [9-15], in the present study, we only concentrate on the local-equilibrium scenarios where Eq.(1) is applicable. In fact, for most engineering applications, the local-equilibrium assumption of saturated porous media can work very well [1].

The most serious shortcoming of Guo's [9] (or Guo's-like [10-15]) thermal LB model for heat transfer in porous media is that it can not address an investigated domain where the heat capacitance varies spatially obviously (as shown below, Guo's model fails to reproduce real physical pictures even there is only a small spatial change of heat capacitance within the investigated domain). In Ref.[9] Guo et al. emphasized that, in order to recover the macroscopic governing equation of heat transfer in porous media Eq.(1) exactly, one has to assume $\sigma$ varies extremely slowly in space. According to Eq.(1), one can see that $\sigma$ is equivalent to the heat capacitance of porous media.

Such shortcoming restricts dramatically the applicable range of Guo's model, since to satisfy practical requirements most realistic systems consist of multilayers of porous media where the specific heat capacities of those porous layers differ with each other significantly $[1,18,19]$. Unfortunately, this deficit has not been addressed yet [9-15], although more than a decade has elapsed since Guo and Zhao proposed their model [9]. Either the available SRT-based [9,11-13,15] or MRT-based $[10,14]$ thermal LB models for local-equilibrium heat transfer in porous media, all always suffer from such drawback.

The purpose of the present work is to remedy such serious shortcoming. The rest of the present paper is organized as follow. In Section2, a thermal LB model for local-equilibrium heat transfer in porous media is presented, which can break the limitation of Guo's model in a simple way. Numerical validation for the present model is conducted in Section 3, followed by a conclusion on this work.

\section{LB model for heat transfer in porous media}

In the present work, we take a SRT-based LB model as an example to show how to remedy the aforementioned shortcoming of Guo's (or Guo-like) thermal LB porous media model [9-15] as Guo's original model adopted the SRT approximation. It is very straightforward to establish its MRT-based counterpart in the same way. For example, to improve the MRT-based thermal porous media model proposed by Liu et al. $[10,14]$. 


\subsection{Evolving equation}

The SRT-based LB evolving equation for energy field used in the present study is the same as that in previous publications $[9,11-13]$

$$
g_{j}\left(x_{\alpha}+c e_{j \alpha} \Delta t, t+\Delta t\right)-g_{j}\left(x_{\alpha}, t\right)=-\tau_{T}^{-1}\left[g_{j}\left(x_{\alpha}, t\right)-g_{j}^{(e q)}\left(x_{\alpha}, t\right)\right] .
$$

In Eq. (2) $\tau_{T}$ is the dimensionless relaxation time for the pseudo-particle distribution $g_{j}\left(x_{\alpha}, t\right)$ at space $x_{\alpha}$ and instant $t . c e_{j \alpha}$ denotes the discrete velocities and $\Delta t$ means the time interval in the LB evolving equation.

However, in the present work, to remedy their shortcoming, the equilibrium distribution $g_{j}^{(e q)}$ in Eq.(2) is different from theirs and reads

$$
g_{j}^{(e q)}=\left\{\begin{array}{l}
T\left(\sigma-\sigma_{0}\right)+\omega_{j} T\left(\sigma_{0}+\frac{c e_{j \alpha} u_{\alpha}}{c_{s}^{2}}\right), \quad j=0 \\
\omega_{j} T\left(\sigma_{0}+\frac{c e_{j \alpha} u_{\alpha}}{c_{s}^{2}}\right), \quad j \neq 0
\end{array}\right.
$$

where $\omega_{j}$ represents the weight coefficients. $\sigma_{0}$ is a reference value of $\sigma$ so $\sigma_{0}$ is a constant within the whole investigated domain. The parameter $c_{s}$ satisfies $c_{s}^{2} \delta_{\alpha \beta}=\sum_{j} \omega_{j} c^{2} e_{j \alpha} e_{j \beta}$ [20]. In the present work, the same as Ref. [9], a D2Q5 lattice for two-dimensional problems is used for numerical validation. For three-dimensional investigated domains, one can invoke a D3Q7 lattice [21]. Such choice can save computational cost efficiently, which is crucial for industrial-level simulation, as explained in our previous work [20].

The temperature $T$ is obtained by

$$
T=\frac{\sum_{j} g_{j}}{\sigma}
$$

and the effective thermal diffusivity $\kappa_{m}$ is given by

$$
\kappa_{m}=\sigma_{0}\left(\tau_{T}-1 / 2\right) c_{s}^{2} \Delta t
$$

According to Eq.(5), it is clear that in the present model the effective thermal diffusivity depends on $\sigma_{0}$, rather than $\sigma$ in Guo's (or Guo's-like) model (c.f. Eq.(30) in Ref. [9]). As shown below, it is the key to remedy their shortcoming.

If the investigated domain is homogeneous, namely $\sigma=\sigma_{0}$ within the whole domain, the present model will reduce to Guo's model [9]. Therefore, Guo's model can be regarded as a special case of the present model. 


\subsection{Multiscale expansion and recovered macroscopic equation}

Equation (2) can be expanded in Taylor series as [4]

$$
\Delta t\left(\partial_{t}+c e_{j \alpha} \nabla_{\alpha}\right) g_{j}+\frac{\Delta t^{2}}{2}\left(\partial_{t}+c e_{j \alpha} \nabla_{\alpha}\right)^{2} g_{j}+\frac{1}{\tau_{T}}\left[g_{j}-g_{j}^{(e q)}\right]=O\left(\Delta t^{3}\right) .
$$

Introducing the multiscale expansion $\partial_{t}=\epsilon \partial_{t 1}+\epsilon^{2} \partial_{t 2}, \nabla_{\alpha}=\epsilon \nabla_{\alpha 1}$ and $g_{j}=$ $g_{j}^{(e q)}+\epsilon g_{j}^{(1)}+\epsilon^{2} g_{j}^{(2)}+O\left(\epsilon^{3}\right)[9]$, we can sort Eq. (6) in terms of $\epsilon$ and $\epsilon^{2}$ as

$$
\begin{gathered}
\left(\partial_{t 1}+c e_{j \alpha} \nabla_{\alpha 1}\right) g_{j}^{(e q)}=-\frac{g_{j}^{(1)}}{\Delta t \tau_{T}}+O(\epsilon) . \\
\partial_{t 2} g_{j}^{(e q)}+\left(\partial_{t 1}+c e_{j \alpha} \nabla_{\alpha 1}\right)\left[\left(1-\frac{1}{2 \tau_{T}}\right) g_{j}^{(1)}\right]=-\frac{g_{j}^{(2)}}{\Delta t \tau_{T}}+O\left(\epsilon^{2}\right) .
\end{gathered}
$$

With the symmetry properties of the lattice $\sum_{j} \omega_{j} c e_{j \alpha}=0$ and $\sum_{j} \omega_{j} c e_{j \alpha} c e_{j \beta}=$ $c_{s}^{2} \delta_{\alpha \beta}$ we can obtain

$$
\begin{gathered}
\sum_{j} g_{j}^{(e q)}=\sigma T, \\
\sum_{j} c e_{j \alpha} g_{j}^{(e q)}=T u_{\alpha}, \\
\sum_{j} c e_{j \alpha} c e_{j \beta} g_{j}^{(e q)}=\sigma_{0} T c_{s}^{2} \delta_{\alpha \beta} .
\end{gathered}
$$

Please bear in mind that the second moment of $g^{(e q)}$ (namely Eq.(11)) is different from that of Guo' model[9]. We will discuss it below.

With the aid of Eqs.(9)-(11), as well as $\sum_{j} g_{j}^{(1)}=\sum_{j} g_{j}^{(2)}=0$, the summation of Eqs.(7)-(8) over the discrete direction $e_{j \alpha}$ reads

$$
\begin{gathered}
\partial_{t 1} \sigma T+\nabla_{\alpha 1} T u_{\alpha}=0+O(\epsilon) \\
\partial_{t 2} \sigma T+\nabla_{\alpha 1}\left[c_{s}^{2}\left(\frac{1}{2}-\tau_{T}\right) \Delta t \nabla_{\alpha 1} \sigma_{0} T\right]=0+O\left(\epsilon^{2}\right) .
\end{gathered}
$$

Because $\sigma_{0}$ is a constant across the whole investigated domain, $\nabla_{\alpha 1} \sigma_{0} T=$ $\sigma_{0} \nabla_{\alpha 1} T$. Accordingly Eq.(13) can be re-written as

$$
\partial_{t 2} \sigma T+\nabla_{\alpha 1}\left[\sigma_{0} c_{s}^{2}\left(\frac{1}{2}-\tau_{T}\right) \Delta t \nabla_{\alpha 1} T\right]=0+O\left(\epsilon^{2}\right)
$$

Combining Eqs.(12) and (14), we can obtain the final recovered macroscopic governing equation for temperature field

$$
\partial_{t} \sigma T+\nabla_{\alpha} T u_{\alpha}=\nabla_{\alpha} \kappa_{m} \nabla_{\alpha} T+O\left(\epsilon^{2}\right)
$$


where $\kappa_{m}=\sigma_{0} c_{s}^{2}\left(\tau_{T}-\frac{1}{2}\right) \Delta t$. It is obvious that Eq.(15) can match Eq.(1) exactly without the restriction that $\sigma$ should be vary extremely slow in space, which exists in Guo's (or Guo's-like) model [9-15].

Through an analysis on Ref. [9], one can find out that such restriction stems from that in Guo's model the second moment of the equilibrium distribution $g_{j}^{(e q)}$ depends on $\sigma$, namely

$$
\sum_{j} c e_{j \alpha} c e_{j \beta} g^{(e q)_{j}}=\sigma T c_{s}^{2} \delta_{\alpha \beta}
$$

Accordingly, through the multiscale expansion, Guo's model generates

$$
\partial_{t 2} \sigma T+\nabla_{\alpha 1}\left[c_{s}^{2}\left(\frac{1}{2}-\tau_{T}\right) \Delta t \nabla_{\alpha 1} \sigma T\right]=0+O\left(\epsilon^{2}\right) .
$$

In Eq.(17), to move $\sigma$ outside from the spatial gradient operator $\nabla_{\alpha 1}$ (i.e. $\nabla_{\alpha 1} \sigma T \simeq \sigma \nabla_{\alpha 1} T$ ), one has to assume $\sigma$ vary spatially slightly, namely $\nabla_{\alpha 1} \sigma \approx$ 0 .

\section{Numerical validation}

In order to validate the present model, natural convection in a square cavity filled by porous media is simulated, which is a benchmark test. Figure 1 illustrates the configuration of the square porous cavity. The cavity is filled by two types of porous media with different heat capacitance. Namely, $\sigma_{1}$ and $\sigma_{2}$ may be different. Accordingly, an inclined interface exists between them. For natural convection simulation, fluid flow should be modelled simultaneously. In the present work, the LB model developed in Ref.[8] is adopted for flow field simulation. A grid resolution $100 \times 100$ is employed and $\sigma_{0}=\left(\sigma_{1}+\sigma_{2}\right) / 2$.

Firstly, we set $\sigma_{1}=\sigma_{2}=1$, the porosity $\epsilon=0.4$, the Darcy number $D a=10^{-2}$ and $10^{-4}$, and the Rayleigh number $R a=10^{4}-10^{7}$. Table 1 lists the calculated average Nusselt number $N u$ on the hot wall, compared with those published in Ref.[9]. In the present work the average Nusselt number $N u$ is defined as

$$
N u=-\frac{1}{L} \int_{0}^{L} \frac{\partial T}{\partial x} d y
$$

where $L$ is the length of the side of the cavity. The results demonstrate when $\sigma_{1}=\sigma_{2}$ the present model can generate the same predictions as Guo's.

Then we set $\sigma_{2}=2 \sigma_{1}$. As shown by Eq. (1), $\sigma$ will not affect the temperature distribution when the system achieves its steady status. it is clear that the numerical predictions by Guo's model are completely false, as shown by Fig. 
2 (a). The maximum temperature calculated by Guo's model is even higher than the temperature on the hot wall. It is obvious that such nonphysical internal heating phenomenon is caused by pseudo numerical diffusion around the interface. Meanwhile, the continuity of temperature distribution near the interface is broken. On the contrary, the present model still works well, illustrated by Fig. 2 (b), which is the same as that of $\sigma_{1}=\sigma_{2}=1$ (c.f. Fig.3 in Ref.[9]).

The above numerical comparison demonstrates that Guo's model will be inapplicable even thought there is only a very small spatial change of heat capacitance of porous media. On the contrary, the present model does not suffer from such deficit.

\section{Conclusion}

Heat transfer in porous media is an important topic in thermal science. Numerical simulation has become a powerful tool to deepen our insight into this topic. Due to its simplicity and reliability, the LB model proposed by Guo et al. [9] for heat transfer in porous media has received increasing attention [10-15]. Unfortunately, Guo's model suffers from a serious drawback that it can not address an investigated domain where the heat capacitance of porous media varies spatially obviously. The present work shows Guo's model will break down even though there is a very small fluctuation of heat capacity in the investigated domain. In order to remedy this shortcoming to extend the applicable range of the LB method, in this work we propose a new and simple LB model for heat transfer in porous media. The numerical results demonstrate the advantage and reliability of the present model.

Although in the present study we only take a SRT LB model as an example to show how to address the variation of heat capacitance of porous media, the extension to its MRT counterpart is straightforward [10,14]. It will be considered in our future work.

\section{Acknowledgments}

This work has received funding from the Universidad Carlos III de Madrid, the European Unions Seventh Framework Programme for research, technological development and demonstration under grant agreement No. 600371, el Ministerio de Economa y Competitividad (COFUND2014-51509), el Ministerio de Educacin, cultura y Deporte (CEI-15-17) and Banco Santander. S. 
Chen would also acknowledge the support from the British Newton Alumni Fellowship Scheme.

\section{References}

[1] Ingham DB, Bejan A, Mamut E, Pop I. Emerging technologies and techniques in porous media. 2003 Kluwer, London.

[2] Ingham DB, Pop I. Transport Phenomena in Porous Media. 2005 Elsevier, Oxford.

[3] Bear J. Dynamics of Fluids in Porous Media. 2013 Elsevier, New York.

[4] Succi S. The lattice Boltzmann equation for fluid dynamics and beyond. Oxford: Oxford university press;2001.

[5] Kang Q, Zhang D, Chen S, He X. Lattice Boltzmann simulation of chemical dissolution in porous media. Physical Review E 2002;65: 036318/1-036318/8

[6] Kuwata Y, Suga K. Large eddy simulations of pore-scale turbulent flows in porous media by the lattice Boltzmann method. International Journal of Heat and Fluid Flow 2015;55:143-157

[7] Grucelski A, Pozorski J. Lattice Boltzmann simulations of heat transfer in flow past a cylinder and in simple porous media. International Journal of Heat and Mass Transfer 2015;86: 139-148

[8] Guo Z, Zhao TS. Lattice Boltzmann model for incompressible flows through porous media. Physical Review E 2002;66: 036304/1-036304/9

[9] Guo Z, Zhao TS. A lattice Boltzmann model for convection heat transfer in porous media. Numerical Heat Transfer, Part B 2005; 47: 157-177

[10] Liu Q, He YL. Double multiple-relaxation-time lattice Boltzmann model for solid-liquid phase change with natural convection in porous media. Physica A 2015;438:94-106

[11] Gao D, Chen Z. Lattice Boltzmann simulation of natural convection dominated melting in a rectangular cavity filled with porous media. International Journal of Thermal Science 2011;50: 493-501.

[12] Seta T, Takegoshi E, Okui K. Lattice Boltzmann simulation of natural convection in porous media. Mathematics and Computers in Simulation 2006;72: 195-200

[13] Shokouhmand H, Jam F, Salimpour MR. Simulation of laminar flow and convective heat transfer in conduits filled with porous media using lattice Boltzmann method. International Communications in Heat and Mass Transfer $2009 ; 36: 378-384$. 
[14] Liu Q, He YL, Li Q, Tao WQ. A multiple-relaxation-time lattice Boltzmann model for convection heat transfer in porous media. International Journal of Heat and Mass Transfer 2014;73:761-775

[15] Wang L, Mi J, Guo Z. A modified lattice Bhatnagar-Gross-Krook model for convection heat transfer in porous media. International Journal of Heat and Mass Transfer 2016;94: 269-291

[16] Gao D, Chen Z, Chen L. A thermal lattice Boltzmann model for natural convection in porous media under local thermal non-equilibrium conditions. International Journal of Heat and Mass Transfer 2014;70:979-989

[17] Wang L, Zeng Z, Zhang L, Xie H, Liang G, Lu Y. A lattice Boltzmann model for thermal flows through porous media. Applied Thermal Engineering 2016;108:6675 .

[18] Wu H, Fan J. Study of heat and moisture transfer within multi-layer clothing assemblies consisting of different types of battings. International Journal of Thermal Sciences 2008;47: 641-647

[19] Qin M, Belarbi R, Ait-Mokhtar A, Nilsson L. Coupled heat and moisture transfer in multi-layer building materials. Construction and Building Materials 2009;23: 967-975

[20] Chen S, Liu Z, Zhang C, He Z, Tian Z, Shi B, Zheng C. A novel coupled lattice Boltzmann model for low Mach number combustion simulation. Applied Mathematics and Computation 2007;193 : 266-284.

[21] Chen S, Yang B, Zheng C. A lattice Boltzmann model for heat transfer in heterogeneous media. International Journal of Heat and Mass Transfer 2016;102: 637-644

[22] Rihab H, Moudhaffar N, Sassi BN, Patrick P. Enthalpic lattice Boltzmann formulation for unsteady heat conduction in heterogeneous media. International Journal of Heat and Mass Transfer 2016;100: 728-736 


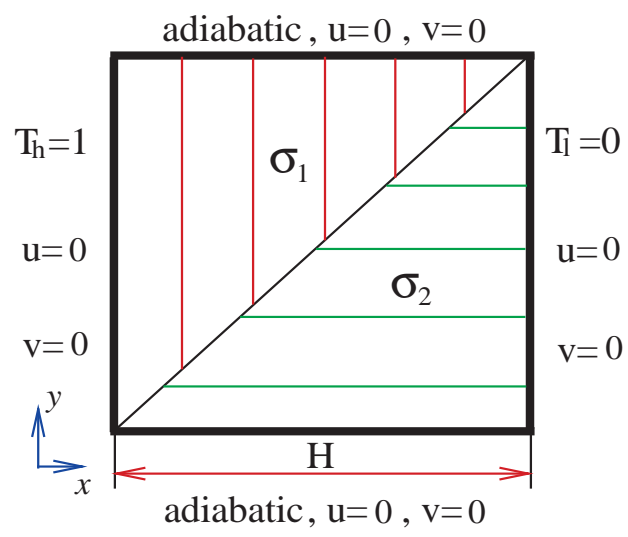

Fig. 1. Schematic configuration of natural convection in a square cavity filled by porous media. 


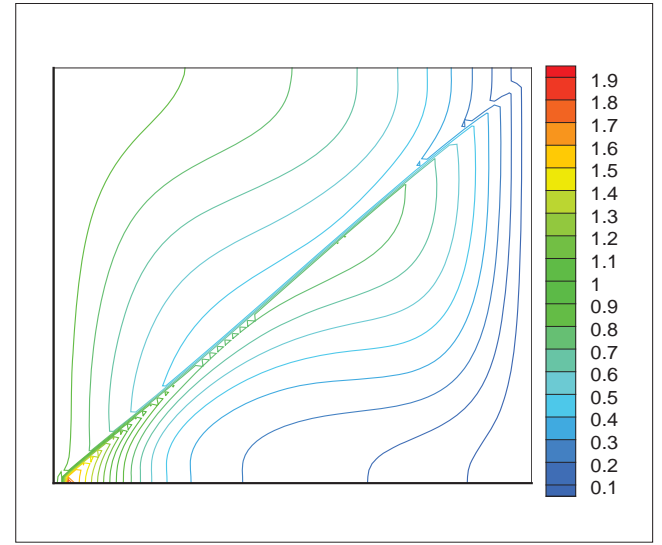

(a)

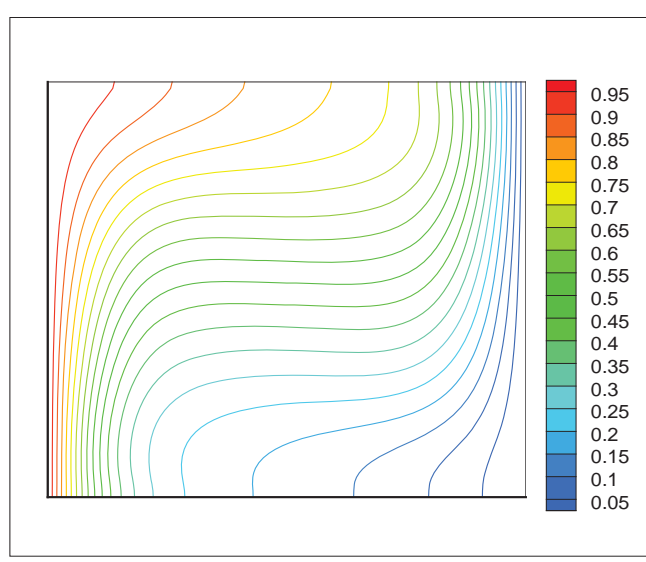

(b)

Fig. 2. Isotherms of natural convection in a porous cavity for $\sigma_{2}=2 \sigma_{1}, \epsilon=0.4$, $D a=10^{-2}$ and $R a=10^{5}$ : Streamlines (a) Guo's model [9] (b) the present model. 
Table 1

Average Nusselt number on the hot wall.

\begin{tabular}{cccc}
\hline$D a$ & $R a$ & present & Ref.[9] \\
\hline $10^{-4}$ & $10^{6}$ & 2.602 & 2.603 \\
& $10^{7}$ & 7.787 & 7.788 \\
\hline $10^{-2}$ & $10^{4}$ & 1.368 & 1.367 \\
& $10^{5}$ & 2.977 & 2.988
\end{tabular}

